\title{
Wenn nicht jetzt, wann dann?
}

\section{Zur Parteienförderung der deutschen Stiftungen in Zeiten der Transition}

\author{
Kristina Weissenbach
}

\section{Kernaussagen}

Trotz des hohen Potentials für das Feld der Parteienförderung und -beratung stehen die deutschen Stiftungen in Zeiten des Systemwandels und der demokratischen Transition vor einer Vielzahl von Herausforderungen. Es gilt wie nun in Nordafrika bereits frühzeitig prodemokratische Einzelpersonen und Gruppierungen zu identifizieren und Dialoge und Netzwerke der oppositionellen Kräfte zu fördern. Für die Ansatz- und Instrumentendefinition der Parteienförderung zum Zeitpunkt der Liberalisierung erscheint es daher angebracht, dass sich die Stiftungen nicht auf die Förderung einer ideologisch nahestehenden (Schwester-)Partei beschränken, sondern den über- und zwischenparteilichen Dialog verbessern und mehrere Parteien unterstützen.

\section{$1 \quad$ Einleitung}

Die Jasmin-Revolution in Tunesien hat in der arabischen Welt eine Demonstrationswelle entfacht. Mit einer Selbstverbrennung als Protest gegen Korruption, Behördenwillkür und Arbeitslosigkeit wurden die Demonstrationen der tunesischen Bevölkerung losgetreten. Innerhalb eines Monats trieben die Protestierenden den Präsidenten Zine el-Abidine Ben Ali aus dem Land. Bemerkenswert ist, dass die politische Massenbewegung weder von einer Partei, einem Oppositionspolitiker noch von der Gewerkschaft formiert wurde sie mobilisierte sich von unten, vielfach kanalisiert über Twitter und soziale Internetnetzwerke: "Thank you Facebook' steht jetzt an der Avenue Habib Bourguiba auf eine Häuserwand gesprüht“ (Melzer, 2011, p.2).

Die Einsetzung der Interimsregierung unter Ministerpräsident Mohamed Ghannouchi, die sich nach wie vor stark aus Vertretern oder Ehemaligen der Regierungspartei RCD zusammensetzte, brachte das Volk nicht zum Ruhen. Nach blutigen Protesten dankte der Übergangspräsident ab und Beji Caid Essebsi wurde zum neuen Präsidenten ernannt und stellte am 7.März 2011 seine neue Regierung vor (Fokus, 2011). Die von der Verfassung vorgegebene Frist von 60 Tagen bis zu einer Neuwahl des Präsidenten wird wohl keine Anwendung finden, die Verfassung selber steht unter Kritik. Ob ihre Revision durch ein neu gewähltes Parlament oder eine Abstimmung der Bevölkerung legitimiert wird, steht noch offen. Der neue Termin für die Präsidentschaftswahl wurde für den Juni 2011 festgelegt. Das gibt den Oppositi- onsparteien, die bislang nur schwach institutionalisiert sind, wichtigen zeitlichen Spielraum um sich zu organisieren.

Auch in Ägypten ging seit dem 25. Januar 2011 das Volk gegen Autokraten auf die Straße - bis schließlich das Militär den Präsidenten Husni Mubarak absetzte. Das Parlament wurde aufgelöst, die vormals regierende National Democratic Party (NDP) ist entscheidungsunfähig und gespalten. Seit dem 11. Februar 2011 steht an der Spitze des Staates nun der Oberste Militärrat, der diese Position mindestens sechs Monate bzw. bis zur Abhaltung freier Wahlen inne haben wird. Ob die Armeespitze nun eine politische Öffnung des Landes einleitet, Verfassungsänderungen und freie Wahlen einführt und einer zivilen Regierung das Zepter übergibt, steht jedoch keinesfalls fest (Asseburg \& Roll, 2011).

Während der Demonstrationen standen auch in Ägypten nicht die etablierten Oppositionsparteien im Zentrum, den Kern bildeten vorwiegend junge Hochschulabsolventen die sich über Facebook organisierten. Wenn es nun um die Frage des Systemwechsels geht und darum, wie das neue politische System ausgestaltet sein wird, sind es jedoch die bestehenden oppositionellen Gruppen, die aufgrund ihrer Institutionalisierung zu den zentralen Akteuren werden:

- säkular orientierte Oppositionsparteien, wie u.a. die national-liberale New Wafd Party unter El-Sayyid el-Baddawi, die sozialistische National Progressive Unionist „Tagammu“" Party, die Nasserist Party oder die liberale Al-Ghad (Carnegie Endowment for International Peace, 2011),

- das noch heterogene Oppositionsbündnis National Association for Change (NAC), das der ehemalige Chef der Internationalen Atomenergiebehörde Muhammed ElBaradei Anfang 2010 gründete (Musharabash, 2011),

- die stark organisierte Muslimbruderschaft, die sich seit Mitte der 1990er Jahre für Parteienpluralismus, Meinungsfreiheit und Gewaltenteilung aussprechen und in der Legislaturperiode 2005-2010 ein Fünftel der Parlamentssitze einnahmen (Asseburg \& Roll, 2011) und

- die Gruppe der Coalition of the Youth of Revolution, die sich jüngst im Kontext der Proteste vor allem aus Facebook-Gruppen (Gruppe „April 6 Youth Movement“, Gruppe „We are all Khaled Said“, Gruppe „Gerechtigkeit und Freiheit“), aus der Jugendkampagne Pro Muhammed ElBaradei, aus der Jugendorganisation der Muslimbruderschaft und der Jugendorganisation der liberalen Democratic Front Party, gründete.

Trotz ihrer Unterschiede zeigen die arabischen Revolutionen gleichermaßen den Willen der oppositionellen Kräfte 
zum Sturz des alten Regimes. Ob sich aus diesem Sturz tatsächlich eine Transition zur Demokratie entwickelt, hängt entscheidend von der Fähigkeit der oppositionellen Kräfte $\mathrm{ab}$, sich zu organisieren und zu institutionalisieren - auch wenn der ,gemeinsame Gegner` nun wegfällt.

\section{Ein Zeitfenster für die Parteienförderung der deutschen Stiftungen?}

Im Kontext der Demokratieförderung betreiben die deutschen parteinahen Stiftungen seit Ende der 60er-Jahre Parteienberatung und -förderung in Transitionsländern. Aus der Umbruchsituation in Tunesien und Ägypten ergibt sich für die deutschen Stiftungen nun ein Zeitfenster, indem junge, reformwillige Akteure vor Ort Wert legen auf Beratung in den Bereichen Verfassungs- und Wahlsystemreform aber vor allem Parteiaufbau und -organisation. Diesen Bedarf hat die Bundesregierung erkannt und unterstützt u.a. die deutschen Stiftungen durch einen neu eingerichteten Demokratisierungsfonds Nordafrika mit einer dreiviertel Millionen Euro. Entwicklungsminister Niebel hebt hierbei die „Kontakte zu den politischen Stiftungen [hervor], die ja teilweise schon 40 Jahre in Ägypten tätig sind und nicht nur mit politischen Parteien ob im oder außerhalb des Parlaments, sondern auch mit vielen gesellschaftlichen Gruppen langjährige Kontakte haben, sodass hier Anknüpfungspunkte sind, wo man Hilfestellung geben kann, wie gründe ich eigentlich eine Partei, wie nominiere ich eigentlich Kandidaten, oder den Verwaltungen helfen kann, wie organisiere ich eine demokratische freie, gleiche und geheime Wahl, was ja ein sehr komplexer Vorgang ist" (Niebel, 2011).

Im Kontext der internationalen Parteienförderer nehmen die deutschen Stiftungen als Quasi-Non-Governmental Organization (QUANGO) eine besondere Position ein: einerseits sind sie sicher staatlich finanziert, andererseits haben sie in ihrer Projektdurchführung de facto freie Hand. Durch ihre langjährige Präsenz vor Ort und das aufgebaute Netzwerk in die unterschiedlichen politischen Lager hinein, verschaffen sich die deutschen parteinahen Stiftungen ein Alleinstellungsmerkmal (Mair, 2000; Weissenbach, 2010).

Viele der deutschen Stiftungen sind seit den 70er Jahren in der Region des nahen und mittleren Osten und Nordafrika aktiv1 und pflegen seit Jahren Kontakt zu oppositionellen Gruppen, führen Gesprächsforen, Workshops, Coachings und Beratungsgespräche durch - entweder in Einpartei- oder Mehrparteienmaßnahmen2. In Tunesien sind mit Ausnahme der Rosa-Luxemburg- und der Heinrich-Böll-Stiftung alle deutschen parteinahen Stiftungen mit Länderbüros oder Projekten vertreten und mit Ausnahme der Rosa-Luxemburg-Stiftung führen alle in Ägypten ein Länderbüro oder zumindest ein Verbindungsbüro.3 Auf Basis dieses Netzwerks üben die Stiftungen vor Ort bereits Beratungsmaßnahmen zu den aktuellen Entwicklungen durch. So bietet die Konrad-AdenauerStiftung in Ägypten im März 2011 Fortbildungen für Nachwuchspolitiker an und veranstaltet Diskussionen und Vorträge zur Zukunft der politischen Parteien in Ägypten (KAS, 2011c)

\section{Implikationen des Systemwandels für die Beratungsansätze der deutschen Stiftungen}

Trotz dieser grundlegend positiven Ausgangsbedingungen für Parteienförderung und -beratung stehen die deutschen Stiftungen in Zeiten des Systemwandels ${ }^{4}$ und der demokratischen Transition vor einer Vielzahl von Herausforderungen die in die Ansatz-, Partner- und Maßnahmendefinition einbezogen werden müssen:

Öffnet sich ein politisches System wie das in Tunesien oder Ägypten tatsächlich dem Liberalisierungsprozess ${ }^{5}$, haben wir es mit einem bunten, heterogenen Strauß an Akteuren zu tun, die Anspruch darauf erheben, über die neuen politischen Institutionen zu befinden: von der Militärführung über reformwillige Einzelpersonen aus den Reihen der bisherigen Regierungsparteien bis hin zu einer Vielzahl oppositioneller Einzelpersonen und Gruppierungen sowie politischen Bewegungen. Eine klare, ideologische Verortung der politischen Kräfte ist zu diesem Zeitpunkt nur im seltensten Fall möglich - bei der Entscheidung für eine oder mehrere Kooperationsparteien ist dies bei den deutschen Stiftungen jedoch ein maßgebliches Auswahlkriterium ${ }^{6}$. Dennoch gilt es in dieser frühen Phase prodemokratische Einzelpersonen und Gruppierungen zu identifizieren und Dialoge und Netzwerke der oppositionellen Kräfte zu fördern. Für die Ansatz- und Instrumentendefinition der Parteienförderung zum Zeitpunkt der Liberalisierung erscheint es daher angebracht, dass sich die Stiftungen nicht auf die Förderung einer ideologisch nahestehenden (Schwester-)Partei beschränken, sondern den über- und zwischenparteilichen Dialog verbessern und mehrere Parteien unterstützen.

Im weiteren Verlauf des Transitionsprozesses bildet sich das neue Parteiensystem meist entlang der Konfliktlinie zwischen dem alten autoritären Regime auf der einen und den Oppositionsparteien auf der anderen Seite. Mit dem Wegfall „des gemeinsamen Feinds“ löst sich diese Konfliktlinie in vielen Fällen jedoch nach kurzer Zeit auf und die zunächst geeinte Opposition zersplittert schnell. Damit gehen meist auch die Beratungsansprechpartner für die Stiftungen verloren, wechseln die Partei oder formieren sich neu. Um diese Spaltung zu verhindern erscheint es bei der Parteienförderung und -beratung angebracht, vor allem die interne und

1 Das Büro der Friedrich-Ebert-Stiftung in Tunis besteht beispielsweise seit 1988, das Büro in Kairo seit 1976. Die Friedrich-Naumann-Stiftung führt ihr Büro in Ägypten seit den 7oer-Jahren.

2 Vgl. zu einer Typologie von Parteienfördermaßnahmen: Burnell, 2001; Weissenbach, 2010.

3 Auch wenn die Stiftungsbüros des Nahen und mittleren Osten teilweise libysche Teilnehmer in Aktivitäten und Beratungsmaßnahmen integrieren, führt keine der deutschen Stiftungen ein Länderbüro in Libyen.

Vgl. FES, 2011a,b; FNS, 2011a,b; HBS, 2011a,b; HSS, 2011a,b; KAS 2011a,b; RLS, 2011.

4 Vgl. zur Abgrenzung der Begrifflichkeiten Systemwandel und Systemwechsel: Hartmann, 1999; Merkel, 1999.

5 Vgl. zur Phaseneinteilung von Transitionsprozessen: Merkel, 1995.

6 Vgl. Interviews der Autorin mit Auslandsbüroleitern von KonradAdenauer- und Friedrich-Ebert-Stiftung sowie mit Stiftungsmitarbeitern im konzeptionellen Bereich Parteienförderung. 
externe Institutionalisierung der Partei ${ }^{7}$ entlang einer klaren gemeinsamen Sach- und Werteorientierung zum Ziel zu haben. ${ }^{8}$

\section{Zur Weiterentwicklung von Parteienförderungsansätzen in Transitionsprozessen}

Trotz ihres großen Potentials für das Feld der Parteienförderung und -beratung arbeiten die Stiftungen in Transitionsländern häufig ad-hoc je nach Partnerlage. Länderspezifische Ansätze oder Beratungsmaßnahmen die sich am Transitionslevel und an der Verfasstheit der Kooperationsparteien vor Ort ausrichten, spielen meist keine Rolle. Für die deutschen Stiftungen in Tunesien und Ägypten hat sich mit dem Sturz der autoritären Machthaber ein Gelegenheitsfenster ergeben, mit ihrer Parteienförderung und -beratung einen Beitrag zum Wechsel in Richtung repräsentatives politisches System zu leisten. Wichtig ist zu verstehen, dass diese Transitionsprozesse im seltensten Fall geradlinig verlaufen, sie unterliegen Rückschlägen oder entwickeln sich in neue Richtungen. Gerade bei solch unsteten Verläufen, sind Parteienförderer auf theoretische Leitbilder angewiesen. Denn bei einer Veränderung der länderspezifischen Rahmenbedingungen geht es darum, einordnen zu können in welcher Verfassung sich die politischen Parteien befinden und welche Rolle sie in der neuen Transitionsphase spielen, um sich als Parteienförderer in der Ziel-, Ansatz- und Maßnahmendefinition neu aufzustellen.

\section{Referenzen}

Asseburg, M., \& Roll, S. (2011). Ägyptens Stunde null? Akteure, Interessen, Szenarien. SWP Aktuell 10, Februar 2011, http://www. swp-berlin.org/fileadmin/contents/products/aktuell/2011A10_ ass rll ks.pdf, 10.03.2011.

Burnell, P. (2001). Promoting Parties and Party Systems in New Democracies: Is there anything the "International Community" can do?. In K. Dowding, J. Hughes \& H. Margetts (Hrsg.), Challenges to Democracy. Ideas, Involvement and Institutions. The PSA Yearbook 2000, (S. 188-204). London: Palgrave.

Carnegie Endowment for International Peace (2011). Carnegie Guide to Egypt's Elections. Political Parties, http://egyptelections.carnegieendowment.org/category/political-parties, 13.03.2011.

FES (2011a). FES International. Naher/mittlerer Osten und Nordafrika: Tunesien, http://www.fes.de/international/nahost/inhalt/ tunes.htm, 11.03.2011.

FES (2011b). FES Tunis. http://www.festunis.org/, 11.03.2011.

FES (2011c). FES International. Naher/mittlerer Osten und Nordafrika: Ägypten. http://www.fes.de/international/nahost/inhalt/ aegyp.htm, 11.03.2011.

FES (2011d). FES Egypt. http://www.fes.org.eg/, 11.03.2011

FNS (2011a): Projekt Ägypten. http://www.freiheit.org/webcom/ show_article.php/_c-204/i.html, 11.03.2011.

FNS (2011) $)$ : FNS Egypt. http://www.fnst-egypt.org/eindex.html, 11.03.2011.

Focus.de (2011). Tunesien. Ministerpräsident Essebsi stellt neue Regierung vor, http://www.focus.de/politik/weitere-meldungen/tunesien-ministerpraesident-essebsi-stellt-neue-regierung-vor aid 606311.html, 10.03.2011.

Hartmann, C. (1999). Externe Faktoren im Demokratisierungsproze $\beta$. Opladen: Leske + Budrich.

HBS (2011). Heinrich Böll Stiftung: Arab Middle East. http://www. boell-ameo.org/, 11.03.2011.

HSS (2011a). Regionen \& Projekte: Tunesien. http://www.hss.de/ index.php?id=1590, 11.03.2011.
HSS (2011b). HSS in den Maghreb. http://www.hssma.org/index. php?lang=de, 11.03.2011

KAS (2011a). Verbindungsbüro Tunesien. http://www.kas.de/tunesien/, 11.03.2011.

KAS (2011b): Auslandsbüro Ägypten. http://www.kas.de/aegypten/, 11.03.2011.

KAS (2011c): Auslandsbüro Ägypten: Veranstaltungen. http://www. kas.de/aegypten/de/events/, 11.03.2011.

Mair, S. (2000). Germany's Stiftungen and Democracy Assistance: Comparative Advantages, New Challenges. In P. Burnell (Hrsg.), Democracy Assistance. International Co-operation for Democratization, (S. 128-149). London\&Portland: Frank Cass.

Melzer, R. (2011). Tunesien in (post)revolutionärer Transformation. Eine Momentaufnahme. In FES (Ed.), Perspektive FES Tunesien. http://library.fes.de/pdf-files/iez/07815.pdf, 10.03.2011.

Merkel, W. (1996). Theorien der Transformation. Die demokratische Konsolidierung postautoritärer Gesellschaften. Politische Vierteljahreszeitschrift. Sonderheft 26/1995. Politische Theorie in der Ära der Transformation, 30-58.

Merkel, W. (1999). Systemtransformation. Eine Einführung in die Theorie und Empirie der Transformationsforschung. Opladen: Leske + Budrich.

Musharabash, Y. (2011). Mohamed ElBaradei. Zauder-Kandidat ohne Basis. http://www.spiegel.de/politik/ausland/o,1518,750129,00. html, 13.03.2011.

Niebel, D. (2011). „Wir werden nicht von außen kolonialisieren“. Interview mit Jasper Barenberg. http://www.dradio.de/dlf/sendungen/interview_dlf/1388679/, 14.03.2011.

Panebianco, A. (1988). Political Parties: Organization \& Power. Cambridge, New York: Cambridge University Press.

Paul, J. (2011). Egypt: The End of Dynastic Republic?. http://www. boell-ameo.org/web/119-549.html, 13.03.2011.

Randall, V. \& Svåsand, L. (2002). Party institutionalization in new democracies. Party Politics, 8(1), 5-29.

Schiller, T. (2011). Tunesien vor dem Wandel?. In KAS (Ed.). Länderbericht Tunesien. http://www.kas.de/tunesien/de/publications/21615/, 11.03.2011.

Spiegel Online (2011). Geflohener Ex-Präsident. Tunesiens Regierung wirft Ben Ali Hochverrat vor, http://www.spiegel.de/politik/ausland/o,1518,749241,00.html, 13.03.2011.

Weissenbach, K. (2010). Political Party Assistance in Transition. The German ,Stiftungen' in sub-Saharan Africa. Democratization, Special Issue Vol. 17, 1225-1249.

Weissenbach, K. (2012). Parteienförderung in der Transition. Eine vergleichende Analyse der deutschen parteinahen Stiftungen FES und KAS in Kenia und Südafrika. Wiesbaden: VS-Verlag für Sozialwissenschaten, i.E..

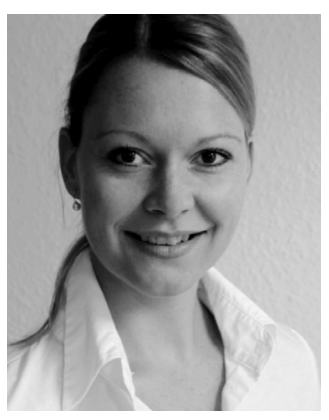

Kristina Weissenbach, M.A., ist wissenschaftliche Mitarbeiterin am Institut für Politikwissenschaft der Universität Duisburg-Essen sowie Mitglied der Forschungsgruppe Regieren an der NRW School of Governance. E-Mail: kristina.weissenbach@uni-duisburg-essen.de.

7 Vgl. zur Definition von Parteieninstitutionalisierung: Panebianco, 1988; Randall \& Svắsand, 2002; Basedau \& Stroh, 2008.

8 Vgl. zu einem Phasenmodell der Parteienförderung und zur Weiterentwicklung von Parteienförderinstrumenten im Kontext von Transitions- und Parteieninstitutionalisierungsprozessen: Weissenbach, 2010, 2012. 